\title{
Methods for injury surveillance in international cricket
}

\author{
J W Orchard, D Newman, R Stretch, W Frost, A Mansingh, A Leipus
}

Br J Sports Med 2005;39:e22 (http://www.bjsportmed.com/cgi/content/full/39/4/e22). doi: 10.1136/bjsm.2004.012732

See end of article for authors' affiliations

......................

Correspondence to: Dr Orchard, CHESM/

School of Physiotherapy, University of Melbourne, Victoria 2033, Australia; johnorchard@msn.com.au

Accepted

21 December 2004
Background: The varying methods of cricket injury surveillance have made direct comparison of published studies in this field impossible.

Methods: A consensus regarding definitions and methods to calculate injury rates in cricket was sought between researchers in this field. This was arrived at through a variety of face to face meetings, email communication, and draft reviews between researchers from six of the major cricket playing nations.

Results: It is recommended that a cricket injury is defined as any injury or other medical condition that either (a) prevents a player from being fully available for selection for a major match or (b) during a major match, causes a player to be unable to bat, bowl, or keep wicket when required by either the rules or the team's captain. Recommended definitions for injury incidence (for matches, training sessions, and seasons) and injury prevalence are also provided. It is proposed that match injury incidence is calculated using a denominator based on a standard time estimated for player exposure in matches, for the purposes of simplicity. This will allow all injury surveillance systems, including those with limited resources, to make calculations according to a standard definition.

Conclusion: The consensus statement presented provides a standard which, if followed, allows meaningful comparison of injury surveillance data from different countries and time periods, which will assist in the possible identification of risk factors for injury in cricket.
C ricket is one of the world's major team sports. Injuries in cricket are common, particularly to fast bowlers. ${ }^{1-7}$ According to van Mechelen et al, ${ }^{8}$ ongoing injury surveillance is a fundamental process behind successful injury prevention. However, successful ongoing injury surveillance in even major sports has proven elusive, partially because of the difficulties in forming consistent injury definitions. ${ }^{9}$ This lack of consensus has severely limited the ability to compare injury rates between countries.

This consensus statement paper attempts to address this problem with respect to international cricket.

\section{METHODS}

Authors of this paper were chosen to represent those Test playing nations where injury surveillance is currently being undertaken or proposed. To minimise the difficulty in forming a consensus, it was decided to limit authorship to one person per country. Where applicable, the official injury surveillance coordinator (as appointed by the national board) was invited to participate. No person invited to join the group of authors refused. The final group are among the most published authors in the cricket injury surveillance literature and represent the countries from which the vast majority of cricket injury surveillance studies have originated.

The consensus statement was arrived at through a variety of face to face meetings, email communication, and draft reviews between researchers. Initially a draft was prepared between the first two listed authors, who have both prepared official reports for their respective countries using similar definitions, via email communication. The other authors were then invited, in the authorship order listed, by being

\footnotetext{
"Methods for injury surveillance in international cricket", a collaboration between cricket authorities in Australia, the United Kingdom, South Africa, New Zealand, the West Indies, and India, is being published simultaneously in the Journal of Science and Medicine in Sport, the British Journal of Sports Medicine, the South African Journal of Sports Medicine, and the New Zealand Journal of Sports Medicine. The paper was concurrently reviewed and edited by the Journal of Science and Medicine in Sport and the British Journal of Sports Medicine.
}

asked to review a draft of the paper and to recommend any revisions. In particular, the authors were asked to make sure that the recommended methods were applicable to their country (or group of countries, in the case of the West Indies), as all nations vary slightly in the scheduling of their home and away cricket matches. The authors would like to acknowledge the reviewers of this paper, as a substantial number of helpful suggestions were made during the review process.

\section{RESULTS}

The following consensus statement was achieved.

\section{Definition of what constitutes an injury}

It is recommended that a cricket injury (or "significant" injury for surveillance purposes) is defined as: any injury or other medical condition that either $(a)$ prevents a player from being fully available for selection for a major match or $(b)$ during a major match, causes a player to be unable to bat, bowl, or keep wicket when required by either the rules or the team's captain.

Notes on this definition:

(1) A player is not fully available for selection if he is injured, and as a result of this injury is only available for selection in a limited capacity. An example is an all-rounder who has an injury that prevents him/her from bowling, but is available to be selected as a batsman only. If this player is not selected in this scenario, he is considered missing through injury rather than non-selection.

(2) A player who is unavailable for selection for injury prevention reasons, but who would be fully fit to play an entire game, is not considered to be suffering a significant injury. This may occur, for example, where a player and/or coach considers that a bowler has bowled too many overs recently and would be at excessive risk of injury if he was selected in a game. In this situation the player is considered rested-that is, not selected for reasons other than through injury. 
(3) With respect to injury surveillance in first class cricket, a "major" match is a Test match, a One Day International, a first class domestic match, or a domestic one day match.

(4) A player who is forced through injury or illness to retire hurt from batting, bat with a runner, or who is unable to finish bowling an over is considered to have suffered a significant injury.

(5) The definition component "...unable to bat, bowl or keep wicket when required by either the rules or the team's captain" is somewhat subjective, but it is expected that it will be applied in a reliable manner. A batsman who cannot bat in his/her usual position, a regular wicketkeeper who must relinquish the gloves, and a bowler who is unable to bowl his/her usual complement of overs are the typical applications of this clause. However, a fielder (other than the wicketkeeper) who is replaced by the 12th man for his fielding tasks only (but who is able to bat and bowl fully when required) is not considered to have suffered a significant injury.

The definition of an injury presented is limited, and is designed to be limited in such a way that all teams using these methods will apply it equally. If teams wish to survey injuries more extensively, they are permitted to define injuries at two levels. A significant injury can be defined in the fashion advised, with this definition used for comparison with other surveillance systems using the same definition. A broader definition, for example, "any medical condition that presents to medical staff, which affects a player's ability to perform during a match or training" can be made to capture a greater number of injuries for the purposes of a specific single study, although this definition is not recommended for comparing results between surveillance systems.

\section{Definition of injury recovery and injury recurrence}

An injury is considered recovered once a player has returned to full (unrestricted) participation in at least one match (of any type or grade).

A recurrent injury is one to the same side and body part and of the same injury type as an injury that previously qualified as a significant injury earlier in the same season, but which had recovered. An injury that is not a recurrent injury is a new injury. A recurrent injury does not necessarily need to be an identical injury in grade of severity to be a recurrence. The same "injury type" requirement means that a rectus femoris muscle strain after a thigh haematoma would be considered a new injury, but after a previous quadriceps muscle strain on the same side would be considered a recurrence.

\section{Definition of seasons, teams, and survey matches}

Traditionally cricket is played for approximately six months of the year, with this six month period being referred to as a "season". International teams now play cricket for many more than six months of the year, and, although domestic seasons do not last for longer than six months, some domestic players will play two different seasons in different countries during a 12 month period. The months over which a season spans should be defined by each surveillance system, with the suggestion that the northern season generally runs from 1 April until 30 September inclusive, and the southern season generally runs from l October until 31 March inclusive. Northern seasons are referred to by the year in which they exclusively occur-for example, 2003whereas southern seasons are referred to by both the calendar years that they span-for example, 2003-2004. For tours or competitions that cross over the starting date of a new season, it is suggested that all matches in a common series are deemed to have occurred in the season in which the first major match of the series started. For example, a series of three Test matches that occurred over March 2003-April 2003 (when the defined season finished on March 31) would be considered to be part of the 2002-2003 season if the starting date of the first Test was 31 March or earlier. Therefore the second and third Tests would be part of the 2002-2003 season even if they were played in April. If only warm up matches occurred in March and the first Test started on 1 April, then the entire series would be considered to be part of the 2003 season. For injury surveillance in nations where the cricket season is not played over the time periods April-September or October-March, an alternative six month period can be defined as a season.

With respect to international injury surveillance, teams are divided into international teams-for example, England, Australia, South Africa, West Indies-and domestic first class teams-for example, Yorkshire, Queensland, Natal, Jamaica. Other non-first class teams-for example, 2nd XI teams for a county, state, or province, under age teams-are not covered by the scope of these definitions. It is noted that the domestic first class teams playing in the West Indies actually represent distinct nations-for example, Jamaica.

Matches are either two innings matches (generally played over three or more days) or one innings matches (table 1). Effectively, almost all one innings matches are played on one day with limited overs per team. One innings matches played over two days without limited overs are possible but do not attract first class or List A status (List A includes one day matches between first class teams, which are not considered "first class" matches), so are not relevant to first class definitions.

For national teams, a major match is a Test match or a One Day International. For first class domestic teams, a major

Table 1 Schedule of matches

\begin{tabular}{lll}
\hline & Two innings match & $\begin{array}{c}\text { One innings (limited over) } \\
\text { match }\end{array}$ \\
\hline $\begin{array}{l}\text { Match between national } \\
\text { teams }\end{array}$ & Test match* & One Day International \\
$\begin{array}{l}\text { Match between non- } \\
\text { class status) teams from }\end{array}$ & Domestic one day match* \\
$\begin{array}{l}\text { the same country } \\
\text { Other match between two } \\
\text { first class teams (eg tour } \\
\text { match between an } \\
\text { international team and a }\end{array}$ & Other List A one day match \\
domestic team) & Other first class match & \\
\hline *Major matches for first class teams. & & \\
\end{tabular}


Table 2 Exposure (player hours per team) for common match types

\begin{tabular}{lllll}
\hline Type of match & $\begin{array}{l}\text { Overs } \\
\text { scheduled }\end{array}$ & $\begin{array}{l}\text { Players per } \\
\text { team }\end{array}$ & $\begin{array}{l}\text { Designated } \\
\text { hours of play }\end{array}$ & $\begin{array}{l}\text { Total player } \\
\text { hours of exposure } \\
\text { per team }\end{array}$ \\
\hline $\begin{array}{l}\text { One day } 50 \text { overs per side } \\
\text { One day } 40 \text { overs per side }\end{array}$ & 100 & 6.5 & 6.7 & 43.3 \\
$\begin{array}{l}\text { One day } 20 \text { overs per side } \\
\text { First class match played over } 3 \text { days }\end{array}$ & 80 & 6.5 & 5.3 & 34.7 \\
(90 overs scheduled per day) & 40 & 6.5 & 2.7 & 17.3 \\
First class match played over 4 days & 270 & 6.5 & 18 & 117 \\
(90 overs per day) & 360 & 6.5 & 24 & 156 \\
First class match played over 5 days & 450 & 6.5 & 30 & 195 \\
(90 overs per day) & & & 28 & 182 \\
First class match played over 4 days & 420 & 6.5 & 28 & \\
\hline (105 overs per day) & & & & \\
\hline
\end{tabular}

match is a first class domestic match or a domestic one day match. Matches between a national team and a domestic team, although attracting first class status, are not considered to be major matches. For injury surveillance systems involving teams that are not of first class status-for example, second XI teams-a different definition of a major match must be made.

\section{Definition of surveillance cohort}

To determine injury incidence and prevalence, a cohort (population) of players to follow must be determined in advance. Therefore injury rates will consider those players who become injured as well as those who avoid injury.

The cohort to be followed for a given team should be referred to as the "squad". A team consists of 12 players ( 11 active players and the 12th man) whereas a squad for a team contains a varied number of players. The squad to be followed can consist of any number of players, although for comparative purposes the "standard" squad size is considered to be 25 players. This number is chosen arbitrarily but is necessary to compare seasonal injury incidence between squads of different sizes.

A squad (cohort) should be chosen for surveillance purposes at the start of the season. The squad is easy to choose if the team contracts players, as all players with a contract can be considered squad members and those without a contract are not included. However, players may need to be added if they are chosen to play for the team from outside the initial squad.

The definition of a squad member for a team may be varied, but an example is as follows:

(1) Any player under contract to the team in question.

(2) Any other player who plays in the team first XI (not including 12th man) or tours overseas with the team, from the time of his/her first game (or the first tour match) until the end of the cricket year or season (when a new round of contracts are awarded).

If a player retires from the squad during the season under survey, he should be considered unavailable through injury for the rest of the season if the retirement was caused by injury or illness, or unavailable for other reasons, if the retirement was for non-injury reasons.

For the purposes of comparing bowlers with batsmen, all players should have their position designated before the start of the season (or before their commencement with the squad). If the definition of a bowler required a certain number of overs to be bowled during the season under survey, then an injured bowler may mistakenly be considered to be a batsman. A bowler is defined at the start of each season as a player who averaged more than five overs bowled in matches played during any of the previous two seasons. As a result of this definition, most "part time" bowlers will be defined as "bowlers". It would be possible to further subdivide bowlers into part time and full time depending on workload.

Non-bowlers can be subdivided into "wicketkeepers" and "batsmen", based on whether they kept wicket in at least $50 \%$ of games played during each season.

Bowlers can be rated as "fast", "fast-medium", "medium" or "slow/spin" according to player profiles listed by Wisden Cricinfo (http://www.cricinfo.com/) which tend to be universally accepted. The major point of contention with respect to these ratings is usually the difference between fast and fast-medium, and therefore these categories can be combined if desired to be considered "pace" bowlers.

The position description of all-rounder is not generally necessary, as all bowlers are required to bat (whether or not they are considered all-rounders). If bowlers are to be split into categories of all-rounders and specialist bowlers, it is suggested that an all-rounder is defined by the cutoff level of a batting average of 25 or greater. Alternatively, bowlers can be subdivided into part time and full time based on average workload.

\section{Presentation of injury rates}

If exposure time is unavailable, descriptive statistics may be presented. However, it is encouraged that records of exposure time will be kept, and therefore two major types of rates should be calculated, injury incidence and injury prevalence.

\section{Calculation of injury incidence}

Injury incidence analyses the number of new injuries (or new plus recurrent) occurring over a given time period, and should be measured in either or all of the following major formats.

\section{Match injury incidence}

Match incidence considers only those injuries occurring during major matches. This can be calculated in two different types of unit (with a time based denominator for injuries overall and with a delivery based denominator when considering batting or bowling injuries separately). Calculation of match incidence in total, with a time based
denominator

The numerator should be number of injuries, and can include either new injuries or injuries in total (new plus recurrent). The denominator should be number of player hours, with the exposure considered to be 43.333 player hours per team per day for days where 100 overs are scheduled. The exposure of 


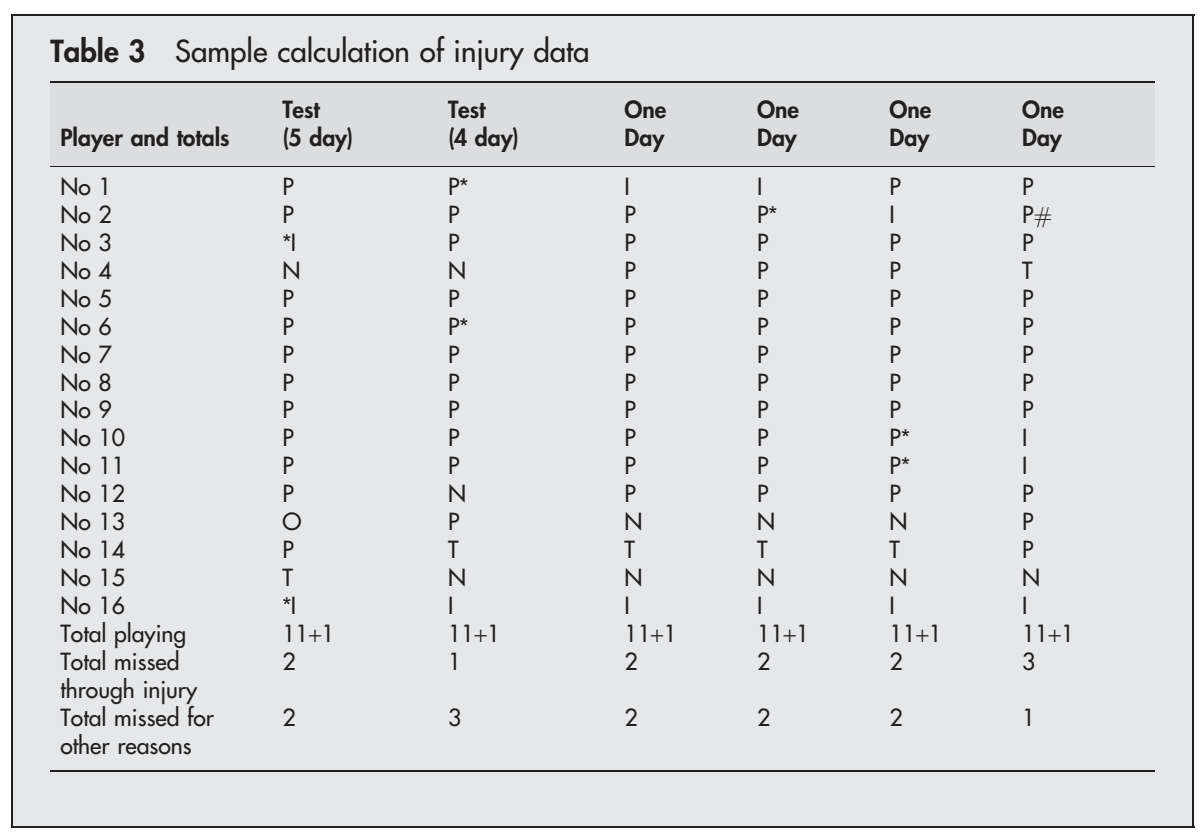

player hours in each day should be factored up or down where more or less than 100 overs of play are scheduled, with a rate of 15 overs per hour assumed. For a "standard" Test or other first class match day with 90 overs scheduled, it is considered that there will be 39 player hours per team per day actually played. These "standard" figures correspond to six hours of play scheduled for first class cricket and 6.667 hours for one day matches (based on a rate of 15 overs bowled per hour). The average number of players exposed is considered to be 13 per two teams or 6.5 per team (at any given time there are 13 players exposed to injury, 11 from the fielding team and two from the batting team). Table 2 lists the exposures to be designated for the most common types of matches. The use of these standard figures does not take into account occasions when matches are shortened by an early finish or lengthened to make up for slow over rates. However, when entire days of play are lost (through a shortened match or adverse weather) this should be accounted for in exposure-for example, a Test match that has only three days of play should be considered 117 player hours per team.

Calculation of batting and/or bowling match incidence, with a delivery based denominator (where delivery information is available)

The numerator should be number of batting injuries and/or number of bowling injuries, and can include either new injuries or injuries in total (new plus recurrent). The denominator for bowling match injuries should be overs bowled, with a preferred unit of injuries per 1000 overs bowled. The denominator for batting match injuries should be deliveries faced, with a preferred unit of injuries per 10000 balls faced. Although this may seem inconsistent to use overs (six balls) in the denominator for bowlers and balls in the denominator for batsmen, this is how scoresheets are maintained in cricket (bowling records indicate overs bowled whereas batting records indicate balls faced). A previous study of bowling injuries has used injuries per 1000 balls bowled, ${ }^{2}$ which can easily be converted into injuries per 1000 overs bowled by multiplying by six.

Because of the agreed definition of a significant injury, it is not recommended that a specific incidence is calculated for fielding, other than wicketkeeping, injuries.

\section{Training injury incidence}

Training incidence can be separately measured from match incidence, although measuring exposure is extremely difficult. However, there is a perceived benefit in measuring bowling workload at training because many bowling injuries are likely to be related to overall (match plus training) workload. ${ }^{10}$ Bowling injury incidence overall (match and training) can be measured in units of injuries per 1000 overs bowled or injuries per 1000 balls bowled if this information is available. An alternative unit that may be developed is bowling injuries per 100 days of bowling exposure, as a recent study has suggested that the number of days (match and training) on which a bowler bowls (overall) may be more important than the total overs bowled. ${ }^{10}$ This unit may also overcome the difficulty in measuring exact workload at training, as it is far easier to record the number of training sessions-that is, one net session or one day where a player bowls in a match = one day of bowling exposure.

\section{Seasonal injury incidence}

Seasonal incidence considers the number of defined injuries occurring per squad per season. This can take into account gradual onset injuries, training injuries, and match injuries in the one measurement. A squad is defined as 25 players, and a season is defined as 60 days of scheduled match play. Smaller or larger squads and longer or shorter seasons should have the incidence adjusted so that rates between different squads and years can be compared. The recommended unit of measurement is injuries per squad per season, with a "squad season" being defined as $1500(25 \times 60)$ player days.

\section{Calculation of injury prevalence}

Injury prevalence considers the average number of squad members not available for selection through injury or illness for each match divided by the total number of squad members. Injury prevalence should be expressed as a percentage, representing the percentage of players missing through injury on average for that team for the season in question. It is calculated using the numerator of "missed player games", with a denominator of number of games multiplied by squad members. An injury prevalence figure of, say, $12 \%$, indicates that for the time period under survey, on 
average $12 \%$ of players were unavailable for selection because of injury or illness.

Injury prevalence should be separately calculated for the different types of cricket (one day matches, three, four, and five day matches), and, when a combined injury prevalence figure is derived for a team for a season, the units should be converted from missed player games into missed player days, so, for example, that each Test match contributes more to overall injury prevalence than each one day match.

The injury surveillance coordinator should keep records of all matches played by squad members and ensure that each team provides an explanation to the survey whenever one of their players was not selected. The common reasons for missing games (with summary codes) are:

- I - injury;

- $\mathrm{U}$ - unavailable due to national team commitments (for domestic squads);

- $\mathrm{T}$ - selected as 12th man;

- $\mathrm{N}$ - not selected (including when rested);

- O - not available for other reasons-for example, suspended or personal reasons.

Example calculations are shown in table 3 for a hypothetical team with 16 players playing a short season (series of six matches).

From table 3, there were seven new injuries reported $\left({ }^{*}\right)$ among this squad of 16 players over this Test and One Day series of six matches. Two injuries occurred in training before the first Test, two occurred during the second Test (one causing the player to retire hurt but not miss a match), and three occurred during the One Day series. There was one recurrent injury (\#) in the One Day series.

Match incidence:

Test Match series match incidence $=2$ new injuries $/(156+$ 195 player hours for the 4 and 5 day Tests respectively)

$=56.98$ injuries per 10000 player hours

One Day series match incidence $=3$ new injuries $/(4$ matches $\times 43.33$ player hours scheduled per match)

$=173.1$ new injuries per 10000 player hours and/or

$=4$ total $($ new + recurrent $)$ injuries $/(4$ matches $\times 43.33$ player hours scheduled per match)

$=230.8$ total injuries per 10000 player hours

Seasonal incidence:

Seasonal incidence $=7$ new injuries $\times(60$ days $\times 25$ players $) /(13$ days played $\times 16$ players in this squad $)$

$=50.5$ injuries per squad per season

The standard squad season is 25 players scheduled for 60 days of cricket. To calculate a seasonal incidence from this short series involving a small squad, the rate is multiplied by the standard squad size and season length and divided by the actual exposure.

Injury prevalence:

Test match injury prevalence rate $=3$ missed player games $/ 32$ exposed player games

$=9.4 \%$

One Day injury prevalence rate $=9$ missed player games/ 64 exposed player games

$=14.1 \%$

Combined injury prevalence rate $=(2 \times 5)+(1 \times 4)$

$=14$ missed Test days +9 missed One Day matches

$=23$ missed player days $/ 208$ exposed player days

$=11.1 \%$

\section{Injury categories and injury classification}

Tabulation by injury category is encouraged. Depending on level of diagnostic accuracy and space this can be done at any or all of the four levels suggested in table 4. For specific diagnosis, use of the cricket specific modification of the OSICS system is freely encouraged. ${ }^{11}$ OSICS is available for download at: http://www.injuryupdate.com.au/research/ OSICS.htm.

\section{Information that should be collected by an injury surveillance system}

Items that should be included on an injury surveillance form (paper, spreadsheet, or database) are listed below:

Details for each injury recorded:

(1) Player name

(2) Player details - for example, date of birth, bowling type

(3) Injury diagnosis (including code and body region)

(4) Injury side (left/right/bilateral/not applicable)

(5) New injury/recurrence

(6) Time of onset (match/training/other/gradual) including match details

(7) Activity of onset (batting/bowling/fielding/gradual), including fielding position

(8) Date of onset

(9) Mechanism description (if available)

(10) Qualification as a significant injury

(11) Details of any surgery required or any other major treatment (if relevant)

Details for player exposure:

(1) Player participation in each major match

(2) Reasons for not participating for all squad members not playing - that is, playing at another level, injured, not available for another reason, not selected

(3) Number of overs bowled in each innings (for all players who bowled)

(4) Number of deliveries faced in each innings (for all players who batted)

(5) Eventual length of the match (in days actually played)

It is preferable to use a database to store injury information, although spreadsheets and other record keeping formats are alternatives. ${ }^{12}$ It is noted that, in all international matches, a match referee is present who must prepare a match report. For the purposes of assisting international injury surveillance, this report could include all injury interruptions to the game (players retiring hurt, batting with a runner, being unable to finish bowling an over, or relinquishing the wicketkeeping gloves).

\section{DISCUSSION}

It is worth commenting on the aspects of the definitions on which it was most difficult for us to reach agreement.

The definition of a significant injury is limited and obviously does not include all occurrences of injuries. However, a broader definition than the one provided-for example, any condition presenting to medical staff-is likely to be subject to a major bias and is therefore unsuitable for use when comparing different teams and/or countries. ${ }^{13}$ The major bias is that the injury rate according to this definition will vary substantially according to the accessibility of medical staff. A team that has medical staff present at every training session-for example, a team physiotherapist-will almost certainly report a higher rate of injuries (according to this definition) than a team in which players must travel to consult medical staff. Therefore the limited definition proposed (of an injury or significant injury) should be the 
Table 4 Injury categories for data tabulation

\begin{tabular}{|c|c|c|c|}
\hline Level 1 & Level 2 & Level 3 & Level 4 \\
\hline \multirow[t]{2}{*}{ Head and neck } & Head and facial & $\begin{array}{l}\text { Fractured facial bones } \\
\text { Other head and facial injuries }\end{array}$ & $\begin{array}{l}\text { Specific diagnoses } \\
\text { Specific diagnoses }\end{array}$ \\
\hline & Neck & Neck injuries & Specific diagnoses \\
\hline \multirow[t]{6}{*}{ Upper limb } & Shoulder & Shoulder tendon injuries & Specific diagnoses \\
\hline & & Other shoulder injuries & Specific diagnoses \\
\hline & Elbow/arm & Arm/forearm fractures & Specific diagnoses \\
\hline & & Other elbow/arm injuries & Specific diagnoses \\
\hline & Wrist and hand & Wrist and hand fractures & Specific diagnoses \\
\hline & & Other wrist and hand injuries & Specific diagnoses \\
\hline \multirow[t]{4}{*}{ Trunk and back } & Trunk & Side and abdominal strains & Specific diagnoses \\
\hline & & Other trunk injuries & Specific diagnoses \\
\hline & Back & Lumbar stress fractures & Specific diagnoses \\
\hline & & Other lumbar injuries & Specific diagnoses \\
\hline \multirow[t]{8}{*}{ Lower limb } & Groin, buttock and thigh & Groin and hip injuries & Specific diagnoses \\
\hline & & Thigh and hamstring muscle strains & Specific diagnoses \\
\hline & & Buttock and other thigh injuries & Specific diagnoses \\
\hline & Knee & Knee cartilage injuries & Specific diagnoses \\
\hline & & Other knee injuries & Specific diagnoses \\
\hline & Shin, foot and ankle & Shin and foot stress fractures & Specific diagnoses \\
\hline & & Ankle and foot sprains & Specific diagnoses \\
\hline & & Other shin, foot and ankle injuries & Specific diagnoses \\
\hline \multirow[t]{2}{*}{ Illness } & Illness & Heat related illness & Specific diagnoses \\
\hline & & Other medical illness & Specific diagnoses \\
\hline
\end{tabular}

one used for highlighted reporting of injury rates-for example, in the abstract-and comparison with other studies.

In terms of the limits provided within the definition of a significant injury, the decision to include those injuries that prevent bowling, batting, and wicketkeeping but not those that limit fielding (other than wicketkeeping) was again made with the aim of consistency among different teams. Because batsmen, bowlers, and wicketkeepers cannot be legally replaced in these roles by the 12 th man, it was felt that there would be a good level of consistency among various teams for the replacement of players in these roles. However, as the 12th man can legally field (in a non-wicketkeeping position) for any player, and some teams take advantage of these substitutions more than others (and at times when it is not absolutely necessary), it was decided not to include being unable to field as part of the injury definition.

The group had difficulty in deciding how accurate calculations should be when determining the exposure for match injuries, and this was the one area of the consensus statement where opinions originally differed strongly. The most accurate method possible would involve counting every ball bowled and faced, and every minute of play. However, it was felt that not all teams (even at the international and first class levels) would have the resources available to easily do this. We decided that specific batting and bowling injury incidence in matches should be calculated based on exact exposure in terms of number of deliveries, and that, where possible, teams should be encouraged to keep records of this amount of exposure. It was agreed that the "headline" match injury incidence rate should be in a time based unit (of injuries per 10000 player hours) but that, owing to the nature of cricket, calculating the exact number of players on the ground for each team and their exact amount of time exposed to injury was fraught with difficulty. Our final decision to have a standard "estimate" of exposure of player hours for each type of standard match (as listed in table 2) was a concession to simplicity, with the aim of encouraging as many studies as possible to use the same definition (particularly those that are not given great financial support). A suggested method for calculating exposure more accurately would be to add minutes batted per team (multiplied by 2 for each batsman on the field) to minutes bowled per team (multiplied by 11 for the bowler and each fielder) to assess total player minutes of exposure. It is possible that the "time estimate" definition supplied will be revised in the future to a method that calculates specific exposure more accurately if it can be shown that $(a)$ it would make a significant difference to the final results of studies and $(b)$ the vast majority of surveillance systems around the world would have the resources to calculate the exposure more accurately.

Choosing the number of players exposed to injury at any given time as 13 for the two teams (6.5 per team) is controversial, but this was done to achieve consistency with other sports. Generally most team sports have a set number of players on the field and a bench of reserves. The number of players exposed in other sports is generally considered to be the number present on the field rather than the total number of players (including the interchange bench). For a sport such as cricket, it is problematic that the two teams do not have an equal number of players on the field at any given time (11 fielding and two batting). An argument can be made that potentially some teams may spend more time batting than others and that exact exposure times for each activity should be calculated because of this. For a single game, or even a single Test series, this imbalance of exposure may mean that the "calculated" exposure of players would be different from the "real" exposure. However, it is assumed that, over a long period of time, teams will spend close to $50 \%$ of their playing time in the field and $50 \%$ of their playing time batting. For superior teams, whose batsmen tend to bat for longer (on average) than weaker teams, their batting exposure will be reduced by those occasions in which the captain declares, or by those occasions where the team wins a match by more than an innings and therefore does not need to bat a second time. A further objection may be that very occasionally, when an injured batsman is batting with a runner, there are actually 14 players on the field. This is not worth taking into account because of its rarity and also because technically the two players are sharing the duties of one player (one playing the ball, the other the running between the wickets) and therefore the injury exposure is not really doubled.

When there was any dispute among authors about a proposed definition, we generally decided to err on the side of definitions that will be adhered to by as many researchers as possible. If the onerous task were added forcing all researchers to attempt to calculate exact fielding and batting 
What is already known on this topic

Various methods of cricket injury surveillance are used, making direct comparison of published studies in this field impossible.

times, it would be a major disincentive to compliance. Even if there is a long term small systematic error introduced by assuming a 50/50 split between batting and fielding, if the error is made consistently by all researchers then it will have far less of a problematic effect than if certain nations refused to follow the injury definitions because the task was too difficult.

The definition provided for injury prevalence differs somewhat from the concepts of injury prevalence in traditional (non-sporting) epidemiology. It is more similar to the concept of "point prevalence" than "period prevalence", although it is actually a prevalence rate. ${ }^{13}$ Compared with sport, life in general is not divided into matches and training sessions. From both performance and insurance viewpoints, the concept of a "missed match" is a fundamental one in sport. ${ }^{14}$ We consider it appropriate that injury prevalence focuses on the number of matches for which players are unavailable, which is the best simple measure of the overall impact that injury has had on a team.

The fundamental concept of a "missed player match" is also the rationale behind including medical conditions apparently sustained outside cricket as "injuries". Firstly, any medical condition that impacts on a player's ability to play is important for both the player and team. Therefore, planning to minimise playing time lost from cricket can justifiably include immunisation against infectious diseases (which may or may not be contracted playing sport). In addition, it removes the necessity for a judgment to be made on whether an injury or illness was related to the sport or not, which can sometimes be difficult-for example, a player who is dehydrated partially because of the effects of gastroenteritis and partially because of playing a match in hot conditions. In our experience, the vast majority of causes of missed playing time among cricketers are indeed conditions caused by playing cricket.

The concept of a "major" match was settled on to signify those matches for which all players would generally be trying to achieve selection. Although they have first class status, matches between a touring international side and a local domestic side are often not viewed as important competitively for the international side, hence many players may be "rested" with a minor injury that may normally have allowed them to play. Because of the difficulty in this scenario of deciding whether a player missed through injury or not, these matches were excluded from the definition of a major match. The other matches listed as major matches by contrast are almost always fully competitive.

Even with the common definitions suggested, there will necessarily be structural factors that will affect injury rates depending on the number and type of matches played in each country. As the number of matches played increases, there is very likely to be a corresponding increase in injury prevalence (percentage of players injured at any given time). However, it is unclear whether there would be any consistent effect on injury incidence. If there is an "overuse" threshold that is crossed, then perhaps an increased number of matches would result in higher injury incidence. However, there may be a reverse effect of a corresponding decrease in injury incidence (injuries per 10000 player hours) with more match hours being played, if the greater number of matches leads to a
What this study adds

A standard system is deviced which should allow meaningful comparison of injury surveillance data from different countries and time periods, which will assist in identification of risk factors for injury in cricket.

decreased intensity of play and/or a decrease in training workload.

It was decided to focus the definitions presented on cricket matches between men at first class level, as there are enough similarities between countries to allow this to be done with minimal complexity. In the future, ideally a similar (or expanded) consensus statement can be made to cover definitions specifically for cricket at amateur and junior levels, and for women's cricket. Hopefully these new definitions will share many of those contained in this statement, but will vary to take into account the different structures of the various cricket seasons and length of matches.

We recommend the use of the definitions and methods presented in this consensus statement in all international and first class domestic cricket. This should make future injury surveillance reports directly comparable and hence more informative in recognising trends over time and differences between countries.

\section{Authors' affiliations}

J W Orchard, University of Melbourne, Australia

D Newman, England \& Wales Cricket Board

R Stretch, UPE Academy of Sport, South Africa

W Frost, New Zealand Cricket High Performance Centre

A Mansingh, West Indies Cricket Board

A Leipus, Indian Cricket team

Author disclosures: all authors are either paid employees or consultants at the various cricket boards listed. The first author is the developer of the OSICS classification system, which is a freely available non-profit system.

\section{REFERENCES}

1 Finch CF, Elliott BC, McGrath AC. Measures to prevent cricket injuries. An overview. Sports Med 1999;28:263-72.

2 Gregory P, Batt M, Wallace W. Comparing injuries of spin bowling with fast bowling in young cricketers. Clin J Sport Med 2002;12:107-12.

3 Leary T, White J. Acute injury incidence in professional county club cricket players (1985-1995). Br J Sports Med 2000;34:145-7.

4 Newman D. A prospective study of injuries at first class counties in England and Wales 2001 and 2002 seasons. In: Stretch RA, ed. Second World Congress of Science and Medicine in Cricket. Cape Town, 2003:83-4.

5 Orchard J, James T, Alcott E, et al. Injuries in Australian cricket at first class level 1995/96 to 2000/01. Br J Sports Med 2002;36:270-5

6 Stretch R. Cricket injuries: a longitudinal study of the nature of injuries to South African cricketers. Br J Sports Med 2003;37:250-3.

7 Stretch R. Epidemiology of cricket injuries. International SportMed Journal 2001;2(2).

8 van Mechelen W, Hlobil H, Kemper $\mathrm{H}$. Incidence, severity, aetiology and prevention of sports injuries: a review of concepts. Sports Med 1992; 14:82-99.

9 Ekstrand, J, Karlsson J. Editorial. Scand J Med Sci Sports 2003:147-9.

10 Dennis R, Farhart R, Goumas C, et al. Bowling workload and the risk of injury in elite cricket fast bowlers. J Sci Med Sport 2003;6:359-67.

11 Orchard J. Orchard sports injury classification system (OSICS). In: Bloomfield J, Fricker P, Fitch K, eds. Science and medicine in sport. 2nd ed. Melbourne: Blackwell, 1995:674-81, Current version available at: http:// www.injuryupdate.com.au/research/OSICS.htm.

12 Orchard J, Blood G. How to use databases in sports medicine research. In MacAuley D, Best TM, eds. Evidence-based sports medicine. London: BMJ Books, 2002:29-42.

13 Last JM. A Dictionary of epidemiology, 3rd ed. Oxford: Oxford University Press, 1995: 129

14 Orchard J, Seward H. Epidemiology of injuries in the Australian Football League, seasons 1997-2000. Br J Sports Med 2002;36:39-45. 